BULL. AUSTRAL. MATH. SOC.

VOL. $30(1984), 481-510$.

\title{
AUTHOR INDEX \\ VOLUMES 21 (1980) TO 30 (1984)
}

Vi to Abatangelo

A translation plane of order 81 and its full collineation group

$29 \quad(1984) \quad 19-34$

Hazar Abu-Khuzam

On rings with nil commutator ideal

A commutativity theorem for semiprime rings

23 (1981) 307-311

Hazar Abu-Khuzam and Adil Yaqub

A commutativity theorem for division rings

27 (1983) 221-224

Dennis Acreman

Asymptotic analysis of partition identities [A]*

21 (1980) 43- 46

28 (1983) 299-301

M.E. Adams, V. Koubek and J. Sichler

Pseudocomplemented distributive lattices with small

endomorphism monoids

28 (1983) 305-318

M. Adelman and P.T. Johnstone

Serre classes for toposes

25 (1982) 103-115

A.R. Aggarwal and M.K. Agrawal

On vector subspaces of certain modular forms of given weights: Addendum

25 (1982) 475-479.

M.K. Agrawal

See A.R. Aggarwal

R.A. Ahlip

See K. Gopalsany

Iqbal Ahmad

See V.P. Gupta

Yousef Alavi, Sabra S. Anderson, Gary Chartrand and S.F. Kapoor

On randomly 3-axial graphs

25 (1982) $\quad 187-206$

Ismail A. Amin and Ferenc A. Szász

On extensions of nilpotent torsion rings by semisimple rings

* [A] Abstract of $\mathrm{PhD}$ thesis.

Copyright Clearance Centre, Inc. Serial-fee code: 0004-9727/84 $\$$ A2.00 + 0.00 . 
Dorothy Ann Anderson

Some problems of inference in two-dimensional

distributions [A]

21 (1980) 311-313

Sabra S. Anderson

See Yousef Alavi

Vasil G. Angelov

Some neutral equations with a control parameter

23 (1981) 383-394

V.V. Anh

See P.D. Tuan

V.V. Anh and P.D. Tuan

Meromorphic starlike univalent functions

$30 \quad(1984) \quad 395-410$

Jonathan Arazy

The $K$-functional of certain pairs of rearrangement invariant spaces

$27 \quad$ (1983) 249-257

James Archer

Principal indecomposable modules for some three-

dimensional special linear groups

22 (1980) 439-455

Efraim P. Armendariz and Jae Keol Park

Compressible matrix rings

$30 \quad(1984) \quad 295-298$

Judith A. Ascione

On 3-groups of second maximal class [A]

$21 \quad(1980) \quad 473-474$

J.R. Arkinstall

Minimal requirements for Minkowski's theorem in the plane I

Minimal requirements for Minkowski's theorem in the plane II

$22(1980) \quad 259-274$

$22(1980) \quad 275-283$

Rauno Aulaskari

On the boundary behaviour of Block and normal

functions

$30 \quad(1984) \quad 299-305$

A. Azzam and E. Kreyszig

On solutions of parabolic equations in regions with edges

$22(1980) \quad 219-230$

Frederick Bagemihl

Localization of Patai's theorem on alephs

25 (1982) 143-145

D.D. Bainov

See A.I. Zahariev

Kevin P. Balanda

Almost disjoint families of representing sets [A]

Families of partial functions

27 (1983) $477-479$

28 (1983) $77-90$

E.J. Balder

An extension of the essential supremum concept with applications to normal integrands and multifunctions A general denseness result for relaxed control theory

Józef Banaś and Stanislaw Wedrychowicz

Functional equations occurring in the theory of

delayed differential equations

27 (1983) 83- 89 
Hans-J. Bandelt

Tolerance relations on lattices

23 (1981) 367-381

Kevin Frederick Barrell

Launching and propagation of light in optical

fibres [A]

21 (1980) 307-309

Heinz Bauer

Elliptic differential operators and diffusion

processes

$30 \quad(1984) \quad 219-237$

Benjamin Baums lag

Free products of locally indicable groups with a single relator

John A. Beachy

Weak ideal invariance and orders in Artinian rings

Weak ideal invariance and orders in Artinian rings:

Corrigendum

Howard E. Bell

See Abraham A. Klein

J.A. Belward

Existence of solutions of Oseen type integral

equations

$29(1984) \quad 57-66$

A.F. Bennett and P.E. Kloeden

Viscous motion in an oceanic circulation model

F.E. Bennett and N.S. Mendelsohn

On the spectrum of Stein quasigroups

$29 \quad(1984) \quad 401-404$

23 (1981) 255-264

25 (1982) $317-320$

A.J. Berrick and R.G. Downey

Perfect McLain groups are superperfect

23 (1981) $443-460$

$21 \quad(1980) \quad 47-63$

$29 \quad(1984) \quad 249-257$

Daniel Bertrand and Michel Waldschmidt

On meromorphic functions of one complex variable

having algebraic Laurent coefficients

$24(1981) \quad 247-267$

F. Beukers

Legendre polynomials in irrationality proofs

$22 \quad(1980) \quad 431-438$

G.P. Bhargava

See R.K. Pandey

B.L. Bhatia and S. Rajasekaran

Coefficient estimates for alpha-spiral functions

28 (1983) 319-329

Prabir Bhattacharya

On the normaliser of a group in the Cayley

representation

25 (1982) $81-84$

Grant R. Bigg

Diffraction and trapping of waves by cavities and

slender bodies [A]

27 (1983) 473-474

David Billington

Degree multisets of hypergraphs [A]

$26 \quad(1982) \quad 479-480$

Elizabeth J. Billington

See James M. Fitz-Gerald 
William D. Blair

Commutative rings whose factors have Artinian rings of quotients

28 (1983) 9- 12

J.R. Blake and G.R. Fulford

Force distribution on a slender body close to an interface

$24 \quad(1981) \quad 27-36$

Walter R. Bloom

Factorisation of Lipschitz functions on zero

dimensional groups

$23(1981) \quad 215-225$

Gilles Blum

A note on the central limit theorem for geodesic random walks

$30 \quad(1984) \quad 169-173$

Karol Borsuk and Jerzy Dydak

What is the theory of shape?

J.M. Borwein

An integral characterization of Euclidean space

Moses A. Boudourides

An existence theorem for ordinary differential equations in Banach spaces

A.K. Boyle and R.J. Serven

$\mathrm{Krull}$ dimension and torsion radicals

R.D. Braddock

See J.-Y. Parlange

Rolf Brandl

A covering property of finite groups

On groups with small Engel depth

23 (1981) 227-235

28 (1983) 101-110

J.L. Brenner and James Wiegold

Two-generator groups II

22 (1980) 113-124

Leo Brewin

The Regge calculus in numerical relativity [A]

$30 \quad(1984) \quad 319-320$

Owen J. Brison

On a criterion for the Hall-closure of Fitting classes

23 (1981) 361-365

P. Broadbridge

Classical and quantum quadratic Hamiltonians [A]

27 (1983) $475-476$

C.J.B. Brookes and Howard Smith

A remark on products of locally soluble groups

$30 \quad(1984) \quad 175-177$

Kevin Broughan

Topologies induced by metrics with disconnected range

$25 \quad$ (1982) 133-142

A. Brown

Solutions of period seven for a logistic difference equation

26 (1982) 263-284

James R. Bunch

Stable algorithms for solving symetric and skew-

symmetric systems

26 (1982) 107-119 
David Burns, Gary Chartrand, S.F. Kapoor and Farrokh Saba

Randomly $k$-axial graphs

23 (1981) 143-156

Gregory Butler

Computational approaches to certain problems in the theory of finite groups [A]

$22(1980) \quad 467-468$

Bi 11 Byers

Periodic points and chaos for expanding maps of the interval

24 (1981) $79-83$

Michael Cambern

Isometries of measurable functions

Pilar Cembranos

On Banach spaces of vector valued continuous functions

A. Caranti and C.M. Scoppola

Central commutators

Pietro Cerone

The time dependent net maternity function [A]

$24(1981) \quad 13-26$

28 (1983) $175-186$

$30 \quad(1984) \quad 67-71$

$21 \quad(1980) \quad 315-316$

J. Chabrowski and H.B. Thompson

On the boundary values of the solutions of linear

elliptic equations

Jan Chabrowski and Rudolf Výborný

Maximum principle for non-linear degenerate equations

of the parabolic type

25 (1982) 251-263

G. A. Chandler

Superconvergence of numerical solutions to second kind integral equations [A]

$S$. Chandra and I. Husain

Symetric dual nondifferentiable programs

27 (1983) $1-30$

Gary Chartrand

See Yousef Alavi

See David Burns

M. L. Chaudhry

See D.F. Holman

C.C. Chen

On a characterization of planar graphs

24 (1981) 289-294

Chia Gek-Ling and Lim Chong-Keang

A note on 1-factors of point determining graphs

$25 \quad(1982) \quad 265-270$

Muneo Chō

See Takayuki Furuta

David Choate

Generators of simple algebras

The density of subsequences

Algebras generated by symmetric idempotents

$21(1980) \quad 475-476$

24 (1981) 295-307

K.G. Choo, K.L. Teo and Z.S. Wu

On an optimal control problem involving second order

hyperbolic systems with boundary controls

$\begin{array}{lll}25 & (1982) & 433-440 \\ 29 & (1984) & 145-149 \\ 29 & (1984) & 303-308\end{array}$

27 (1983) 139-148 
N.N. Chourasia

Quasi-similarity orbit of a subclass of compact

operators on a Hilbert space

22 (1980) $85-98$

N.N. Chourasia and P.B. Ramanujan

Paranormal operators on Banach spaces

$21 \quad(1980) \quad 161-168$

Shue-Sum Chow

Finite element error estimates for nonlinear elliptic problems of monotone type [A]

28 (1983) 445-446

King-wah Eric Chu

Deferred correction for the ordinary differential

equation eigenvalue problem

$26 \quad$ (1982) $445-454$

Jen-chung Chuan and Wai-fong Chuan

On the generalized Hadamard product and Jordan-

Hadamard product

$22 \quad(1980) \quad 321-337$

Wai-fong Chuan

See Jen-chung Chuan

David I. Clark

Finite algorithms for linear optimisation problems [A] 24 (1981) 315-316

G.T. Clarke

Semigroup varieties with the amalgamation property [A] 26 (1982) 473-475

Graeme L. Cohen

Generalised quasiperfect numbers [A]

27 (1983) $153-155$

Ian Leslie Collings

Continental shelf dynamics [A]

27 (1983) $157-158$

Ian F. Collins

Boundary value problems in plasticity theory

26 (1982) 121-141

Marston D.E. Conder

Some results on quotients of triangle groups

R.J. Cook

An effective seven cube theorem

30 (1984) $73-90$

$30 \quad(1984) \quad 381-385$

Luis A. Cordero and Manuel de Leon

Prolongations of linear connections to the frame bundle

28 (1983) $367-381$

William H. Cornish

An ordered sheaf representation of subresiduated

lattices

Varieties generated by finite $\mathrm{BCK}$-algebras

Trees as commutative $\mathrm{BCK}$-algebras

22 (1980) 125-132

22 (1980) 411-430

23 (1981) 181-190

William H. Cornish and A.S.A. Noor

Standard elements in a nearlattice

26 (1982) 185-213

Stephen C. Cowin

Adaptive elasticity

26 (1982) $\quad 57-80$

B.D. Craven

Invex functions and constrained local minima

24 (1981) $357-366$ 


\section{Stuart F. Crosbie}

The mathematical modelling of capture-mark-recapture experiments on animal populations [A]

Sándor Csörgö and C.R. Heathcote

Some results concerning symmetric distributions

$\begin{array}{lll}21 & (1980) & 479-480 \\ 25 & (1982) & 327-335 \\ 26 & (1982) & 393-397 \\ 21 & (1980) & 229-236\end{array}$

M.J. Curran

A non-abelian automorphism group with all

automorphisms central

Eispeth Cusack

Normal Fitting classes and Hall subgroups

E.N. Dancer

An implicit function theorem with symmetries and its application to nonlinear eigenvalues

21 (1980) $81-91$

Gregory Bruce Davis

Mathematical modelling of rate-limiting mechanisms of pyritic oxidation in overburden dumps [A]

Brian J. Day

Free finitary algebras on compactly generated spaces

B.J. Day

Coproducts of algebras and derivations on categories

David E. Daykin and Roland Häggkvist

Degrees giving independent edges in a hypergraph

Satya Deo and Dalip Singh Jamwal

Countable sum theorem for locally closed sets

Satya Deo and Subhash Muttepawar

Inverse limit theorem and expansion theorem for cohomological dimension

M. Paul Devasahayam

Monotone method and periodic solution of non linear parabolic boundary value problem for systems

29 (1984) 231-242

K.K. Dewan and N.K. Govil

An inequality for the derivative of self-inversive polynomials

27 (1983) 403-406

P.G. Dixon

Automatic continuity of positive functionals on

topological involution algebras

$29 \quad(1984) \quad 429-430$

$24 \quad(1981) \quad 277-288$

$30 \quad(1984) \quad 153-156$

23 (1981) 103-109

29 (1984) $47-55$

28 (1983) $67-75$

David E. Dobbs

On flat finitely generated ideals

David E. Dobbs and Marco Fontana

Universally incomparable ring-homomorphisms

23 (1981) 265-281

21 (1980) 131-135

29 (1984) 289-302

R.G. Downey

Abstract dependence, recursion theory, and the lattice of recursively enumerable filters [A]

See also A.J. Berrick

27 (1983) 461-464

F. Downton

Rational roulette 
Victor Charles Drastik

Minimum mean squared error estimation [A]

$\begin{array}{lrr}30 & (1984) & 317-318 \\ 28 & (1983) & 53-66 \\ 24 & (1981) & 93-122 \\ 27 & (1983) & 165-183 \\ 24 & (1981) & 133-147\end{array}$

Bau-Sen Du

Are chaotic functions really chaotic

Duong Minh Duc

Differential calculus in Fréchet spaces

Analytic contractive vector fields in Fréchet spaces

Ivo Düntsch

A class of projective Stone algebras

24 (1981) 133-147

Alain Durand

Simultaneous diophantine approximations and Hermite's method

$21 \quad(1980) \quad 463-470$

Jerzy Dydak

See Karol Borsuk

David Easdown

Biordered sets of semigroups [A]

$29 \quad(1984) \quad 425-427$

Howard Paul Edwards

Bayes sequential design procedures: theory and applications [A]

$21 \quad(1980) \quad 157-158$

P.M. Edwards

Eventually regular semigroups

Roger B. Eggleton and Donald K. Skilton

Graphs with eulerian chains

Saber Elaydi

On equicontinuous transformation groups

On locally weakly almost periodic transformation

groups

Transformation groups of strong characteristic 0

Saber Elaydi and Hani R. Farran

Isometries and certain dynamical systems

28 (1983) 23- 38

$29 \quad(1984) \quad 389-399$

$24 \quad(1981) \quad 367-372$

25 (1982) 215-219

27 (1983) 243-248

$30 \quad(1984) \quad 239-246$

L. Erbe

Comparison theorems of Hille-Wintner type for third

order linear differential equations

Trevor Evans and Bernhard Ganter

Varieties with modular subalgebra lattices

$21 \quad(1980) \quad 175-188$

28 (1983) $247-254$

E.J. Farre11

On a class of polynomials associated with the

subgraphs of a graph and its application to chromatic and dichromatic polynomials

26 (1982) 343-354

Hani R. Farran

See Saber Elaydi

Shalom Feigelstock

The additive groups of subdirectly irreducible

rings II

22 (1980) 407-409

A note on subdirectly irreducible rings

$30 \quad(1984) \quad 137-141$ 


\section{J.D. Fenton}

on the numerical solution of convective equations

P.C. Fenton

Entire functions having asymptotic functions

Small subharmonic functions

M.J. Field

Handlebody decompositions for $G$-manifolds

On the structure of a class of equivariant maps

Nigel John Fisher

A quadratic programming algorithm for geophysical gravity inversion and other applications [A]

James M. Fitz-Gerald and Elizabeth J. Billington

Manufacturing sequences for the Economic Lot

Scheduling problem

Patrick Fitzpatrick

Varieties of nilpotent groups of class four [A]

Patrick Fitzpatrick and James Wiegold

Embedding group amalgams

Simon Fitzpatrick

Metric projections and the differentiability of

distance functions

Michael John Flaherty

Time-frequency analysis of speech via modulated splines [A]

J.A. Flanigan

On the distribution of winning moves in random game

trees

Marco Fontana

See David E. Dobbs

Lawrence K. Forbes

Non-linear free-surface flows about blunt bodies [A]

Peter R. Fowler

De Morgan algebras [A]

Maria Fragoulopoulou

Abstract Bochner-Weil-Raikov theorem in topological

algebras

Peter Frankl

On intersecting families of finite sets

Volf Frishling

Controlled Markov processes and related semigroups of operators [A]

G.R. Fulford

See J.R. Blake

Takayuki Furuta

A Hilbert-Schmidt norm inequality associated with the Fuglede-Putnam theorem $\begin{array}{lrr}26 & (1982) & 81-105 \\ 26 & (1983) & 321-328 \\ 29 & (1984) & 67-82 \\ & & \\ 25 & (1982) & 29-36 \\ 26 & (1982) & 161-180\end{array}$

25 (1982) I59-160

24 (1981) $405-431$

$22(1980) \quad 475-476$

24 (1981) 373-379

$22(1980) \quad 291-312$

$26 \quad(1982) \quad 469-470$

24 (1981) 227-237

$25 \quad(1982) \quad 157-158$

25 (1982) 305-307

26 (1982) 39- 44

21 (1980) 363-372

28 (1983) 441-442

25 (1982) 177-185 
Takayuki Furuta and Muneo Chō

Necessary and sufficient conditions for spectral sets

$24 \quad(1981) \quad 349-355$

Jason Gait

Stability in the gaming equation

21 (1980) 207-210

Selvanayagam Ganesalingam

On the mixture maximum likelihood approach to

estimation and clustering [A]

24 (1981) 309-312

M.S. Ganesan

See K.S. Padmanabhan

Bernhard Ganter

See Trevor Evans

B.J. Gardner

Some degeneracy and pathology in non-associative radical theory II

$23(1981) \quad 423-428$

H.M. Gastineau-Hills

Systems of orthogonal designs and quasi Clifford algebras [A]

$24 \quad(1981) \quad 157-158$

David Gauld

The relative Schoenflies theorem

D.B. Gauld, I.L. Reilly and M.K. Vamanamurthy

On normal subspaces

David B. Gauld and M.K. Vamanamurthy

Cardinality of discrete subsets of a topological space

Cardinality of discrete subsets of a topological

space: Corrigendum

Serge Gautier, George Isac and Jean-Paul Penot

Surjectivity of multifunctions under generalized

differentiability assumptions

John Anthony Gear

Wave propagation in density stratified fluids [A]

$22(1980) \quad 249-251$

$23(1981) \quad 1-4$

25 (1982) 99-101

$29(1984) \quad 431$

A. Ghosal

See D.F. Holman

Greg G. Gibbon

Set mappings of unrestricted order

28 (1983) 199-206

Herbert A. Gindler and Jerome A. Goldstein

Dissipative operators and series inequalities

$23(1981) \quad 429-442$

Bevil M. Glover

Generalized convexity in nondifferentiable programing

$30 \quad(1984) \quad 193-218$

Moshe S. Goldberg

Distributive p-algebras and Ockham algebras: a

topological approach [A]

Distributive Ockham algebras: free algebras and

injectivity

22 (1980) $159-160$

24 (1981) 161-203

Jerome A. Goldstein

See Herbert A. Gindler 
Jairo 2. Gonçalves

See Arnaldo Mandel

Heinz H. Gonska

On almost-Hermite-Fejér-interpolation: pointwise estimates

On approximation of continuously differentiable

functions by positive linear operators

On approximation in spaces of continuous functions

25 (1982) 405-423

27 (1983) 73- 81

28 (1983) 411-432

S.J. Goodenough and T.M. Mills

On interpolation polynomials of the Hermite-Fejér type II

23 (1981) 283-291

K. Gopalsamy

Time lags and density dependence in age dependent two species competition

Stability, instability, oscillation and nonoscillation

in scalar integrodifferential systems

Oscillations in linear systems of differential-

difference equations

Some delays do not matter

Linear systems of nonoscillatory differential

equations with delayed arguments

$\begin{array}{lll}25 & (1982) & 271-291 \\ 28 & (1983) & 233-246 \\ 29 & (1984) & 377-387 \\ 30 & (1984) & 91-98 \\ 30 & (1984) & 307-314\end{array}$

K. Gopalsamy and R.A. Ahlip

Time delays in $n$-species competition - I: Global

stability in constant environments

N.K. Govil

See K.K. Dewan

Ivan G. Graham

The numerical solution of Fredholm integral equations of the second kind [A]

$24 \quad(1981) \quad 153-154$

Peter Greim

Hilbert spaces have the Banach-Stone property for

Bochner spaces

27 (1983) 121-128

Peter Alexander Grossman

Polynomial interpolation on universal algebras [A]

27 (1983) 315-317

T.R. Gulati

On converse duality for a nondifferentiable program

B.C. Gupta and P.B. Ramanujan

A note on $d$-symmetric operators

On k-quasihyponormal operators II

V.P. Gupta and Iqbal Ahmad

On starlike functions

27 (1983) 427-441

25 (1982) $71-80$

23 (1981) 471-475

24 (1981) 61- 67

22 (1980) 241-247

Roland Häggkvist

See David E. Daykin

P.R. Halmos

Does mathematics have elements?

25 (1982) 161-175

Eugene P. Hamilton

A new definition of variational derivative
22 (1980) 205-210 
M.J. Hamilton

A finite element approach to molecular vibration [A] 30 (1984) 159-160

Grahame Hardy

Extensions of theorems of Gagliardo and Marcus and

Mizel to Orlicz spaces

Nemitsky operators between Orlicz-Sobolev spaces

$23(1981) \quad 121-138$

30 (1984) 251-269

Brian Hartley, John C. Lennox and Akbar H. Rhemtulla

Cyclically separated groups

$26 \quad(1982) \quad 355-384$

Alan Hartman

Construction and resolution of quadruple systems [A] 23 (1981) 319-320

William F. Hawkins

The mathematical work of John Napier (1550-1617) [A] 26 (1982) 455-468

J.M. Heath

The coupled diffusion of heat and moisture in bulk

wool [A]

27 (1983) 149-150

Phillip Ronald Helm

Linear groups: On non-congruence subgroups and

presentations [A]

$26 \quad(1982) \quad 477-478$

Allison Heard

The solution of the order conditions for general

linear methods [A]

C.R. Heathcote

See Sándo Csörgö

Peter M. Heffernan

Linear location estimators: the dependence of their quality on the shape of the probability density

function, and their robustness [A]

$22(1980)$

319

Joachim Heinze

A remark on a paper of V.K. Srinivasan

$22(1980) \quad 153-154$

J. L. Hickman

A note on the concept of multiset

$22(1980) \quad 211-217$

Peter M. Higgins

A semigroup with an epimorphically embedded subband

Epimorphisms and semigroup varieties [A]

27 (1983) 231-242

29 (1984) 415-417

Howard Hiller

Almost complex structures on four-dimensional complete

intersections

$30 \quad(1984) \quad 143-152$

Jonathan A. Hillman

Trivializing ribbon links by Kirby moves

Polynomials determining Dedekind domains

$21 \quad(1980) \quad 21-28$

29 (1984) 167-175

Leslie J. Hills

Time series in medical research [A]

$30 \quad(1984) \quad 157-158$

Kar1 H. Hofmann

A note on Baire spaces and continuous lattices

21 (1980) 265-279 
D.F. Holman, M.L. Chaudhry and A. Ghosal

Some results for the general bulk service queueing system

$23(1981) \quad 161-179$

Ulrich Hornung

The mathematics of hysteresis

$30 \quad(1984) \quad 271-287$

Janet A. Hoskins, Cheryl E. Praeger and Anne Penfold Street

Twills with bounded float length

28 (1983) 255-281

Guan Aun How

Some classes of monomial groups [A]

$22(1980) \quad 477-478$

R.M. Huggins

See D.J. Scott

G. Huisken

On pendent drops in a capillary tube

28 (1983) 343-354

I. L. Humbers tone

Monadic representability of certain binary relations

$29 \quad(1984) \quad 365-376$

I. Husain

See S. Chandra

$\mathrm{Hu}$ Shaing

See V.K. Srinivasan

George Isac

See Serge Gautier

Brad Jackson and T.D. Parsons

On $r$-regular $r$-connected non-hamiltonian graphs

24 (1981) 205-220

Brad Jackson and Gerhard Ringel

Coloring island maps

29 (1984) 151-165

Dalip Singh Jamwal

See Saty a Deo

Krzysztof Jarosz

A characterization of weak peak sets for function

algebras

$30 \quad(1984) \quad 129-135$

Brian Jefferies

Conditional expectation for operator-valued measures and functions

$30 \quad(1984) \quad 421-429$

N.L. Johnson

Some translation planes constructed by multiple

derivation

23 (1981) 313-315

P.T. Johns tone

See M. Adelman

Mohan Joshi

On the existence of optimal controls in Banach spaces 27 (1983) 395-401

V.N. Joshi

A determinant for rectangular matrices

21 (1980) $137-146$

O.P. Juneja

See M.L. Mogra 
O.P. Juneja and S.M. Shah

Univalent functions with univalent Gelfond-Leontev derivatives

$29 \quad(1984) \quad 329-348$

Philip John Kachoyan

Non-linear effects in laminar flow over wavy surfaces [A]

28 (1983) 303-304

Michael J. Kallaher and Graham Kelly

Line rank 3 affine planes

Gudrun Kalmbach

Automorphism groups of orthomodular lattices

P. Kanniappan and Sundaram M.A. Sastry

A duality theorem for nondifferentiable convex programming with operatorial constraints

G.P. Kapoor and A. Nautiyal

Approximation of entire functions over Carathéodory domains

$25 \quad(1982) \quad 397-403$

29 (1984) 309-313

$22(1980) \quad 145-152$

25 (1982) 221-229

O.P. Kapoor and S.B. Mathur

Some geometric characterizations of inner product spaces

24 (1981) 239-246

S.F. Kapoor

See Yousef Alavi

See David Burns

G. Karpilovsky

On certain group ring problems

21 (1980) 329-350

Stefano Kasangian and Fabio Rossi

Some remarks on symmetry for a monoidal category

Saroop K. Kaul and Dale V. Thompson

On a theorem of Nielsen

23 (1981) 209-214

$24 \quad(1981) \quad 37-47$

G.M. Kelly

A unified treatment of transfinite constructions for

free algebras, free monoids, colimits, associated

sheaves, and so on

On the essentially-algebraic theory generated by a

sketch

Two addenda to the author's 'Transfinite

constructions'

$22(1980) \quad 1-83$

26 (1982) 45- 56

26 (1982) 221-237

Graham Kelly

See Michael J. Kallaher

Nobuyuki Kemoto

A characterization of the existence of a Souslin line 25 (1982) 425-431

P.E. Kenne

Presentations for some direct products of groups

28 (1983) 131-133

Roshdi Khalil

On some Banach space sequences

25 (1982) 231-241

M.A. Khan

A theorem on power-open LCA groups and its consequences 
M.S. Khan

Free products of topological groups with central

amalgamation [A]

$24(1981) \quad 155-156$

M.S. Khan, M. Swaleh and S. Sessa

Fixed point theorems by altering distances between the points

30 (1984) $1-9$

N.M. Khan

Some saturated varieties of semigroups

$27 \quad(1983) \quad 419-425$

Harri Tapio Kiiveri

A unified theory of causal models [A]

$29 \quad(1984) \quad 137-138$

Daniel G. Kildea

Median estimators for regression models - the Brown-

Mood approach [A]

$21(1980) \quad 153$

Ann Chi Kim

Laws in groupoids derived from semigroups

Ann Chi Kim, B.H. Neumann, and A.H. Rhemtulla

More Fibonacci varieties

R. R. King

Relativistic fluid spheres [A]

$26 \quad(1982) \quad 385-392$

22 (1980) 385-395

27 (1983) $465-466$

B.S. Ki ranagi

See G. Prema

Kazuaki Kitahara

On two new classes of locally convex spaces

28 (1983) 383-392

Fuad Kittaneh

On the structure of polynomially normal operators

Thomas Kiventidis

Systèmes dynamiques dans les espaces ultramétriques compacts

Comparaison resultats pour la stabilité pratique

29 (1984) 207-214

$29(1984) \quad 243-248$

Abraham A. Klein, Itzhak Nada, and Howard E. Bell

Some commutativity results for rings

$22 \quad(1980) \quad 285-289$

P.E. Kloeden

See A.F. Bennett

Ronald A. Knight

A characterization of recurrent motions

H. - B. Knoop and B. Stockenberg

On Hermite-Fejér type interpolation

$30 \quad(1984) \quad 11-18$

Mat thew William Knuiman

Combining information in linear and log-linear analyses [A]

Anders Kock

Differential forms with values in groups

P.N. Kokic and N.C. Weber

on the asymptotic behaviour of the jackknife for

stochastic processes

28 (1983) 293-294

25 (1982) $357-386$

28 (1983) $1-4$

28 (1983) 39- 51

27 (1983) 329-337 


\section{Koubek}

See M.E. Adams

E. Kreyszig

See A. Azzam

Vinod Kumar and S.L. Shukla

Certain integrals for classes of $p$-valent meromorphic functions

On $p$-valent starlike functions with reference to the Bernardi integral operator

R.G. Laha and V.K. Rohatgi

Operator semistable probability measures on a Hilbert space

Operator semistable probability measures on a Hilbert space: added in proof

Jane Marie Lake

Raikov systems and abstract harmonic analysis [A]

25 (1982) 85- 97

30 (1984) $37-43$

Edwin Hans van Leeuwen

Spacetime killing tensors in general relativity [A]

$22(1980) \quad 397-406$

22 (1980) $479-480$

27 (1983) 319-320

$24 \quad(1981) \quad 471-474$

G. I. Lehrer

The spherical building and regular semisimple elements

John C. Lennox

See Brian Hartley

Manuel de Leon

See Luis A. Cordero

Pui-Fai Leung

Complete hypersurface of non-positive Ricci curvature

On the codimension of a minimal immersion

27 (1983) 215-219

30 (1984) 431-433

Richard Henry Levingston

Primitive permutation groups containing a cycle of

prime-power length [A]

22 (1980) $315-317$

Mordechai Lewin

On a certain kind of reducible rational fractions

$21 \quad(1980) \quad 321-328$

Lim Chong-Keang

See Chia Gek-Ling

C.H.C. Little

A generalisation of a recent characterisation of

planar graphs

$27 \quad(1983) \quad 225-230$

C.W. Lloyd-Smith

Problems on the distribution of conjugates of

algebraic numbers [A]

On a problem of Favard concerning algebraic integers

25 (1982) 303-304

30 (1984) 111-121

D.A. Lockington

See J.-Y. Parlange

S. L. Loi

Quadratic approximation and its application to

acceleration of convergence [A] 


\section{Lewis Low}

Some lattice point problems [A]

21 (1980) 303-305

J.H. Loxton

A method of Mahler in transcendence theory and some of its applications

$29 \quad(1984) \quad 127-136$

Richard J. Loy

Function spaces on the unit circle

$27 \quad(1983) \quad 107-113$

M.A. Lukas

Regularization of linear operator equations [A]

24 (1981) 313-314

I.G. Macdonald

Numbers of conjugacy classes in some finite classical groups

Hugh MacPherson

Some studies of wave-seabed interactions [A]

$23(1981) \quad 23-48$

Andrzej Magdrecki

Relation between the Shapley value and null group

cohomology

Sadahi ro Maeda

Real hypersurfaces of a complex projective space II

Kurt Mahler

Some suggestions for further research

R.A. Maller

On one-sided boundedness of normed partial sums

An extension of Kesten's generalised law of the

iterated logarithm

\section{J.J. Malone}

p-groups with non-abelian automorphism groups and all automorphisms central

Arnaldo Mandel and Jairo $Z$. Gonçalves

Free $k$-tuples in linear groups

22 (1980) 465-466

Michael Kelly Mara

Empirical Bayes with a changing prior [A]

$28 \quad(1983) \quad 187-197$

$30 \quad(1984) \quad 123-127$

$29 \quad(1984) \quad 101-108$

21 (1980) 373-391

$21 \quad(1980) \quad 393-406$

$29 \quad(1984) \quad 35-37$

$28 \quad(1983) \quad 151-157$

$21 \quad(1980) \quad 151-152$

S.B. Mathur

See O.P. Kapoor

R.M.M. Mattheij

On decoupling of linear recursions

The stability of LU-decompositions of block

tridiagonal matrices

27 (1983) 347-360

29 (1984) 177-205

Rex W. Matthews

Permutation polynomials in one and several

variables [A]

$28 \quad(1983) \quad 287-289$

K. L. McAvaney

Composite graphs with stability index one [A]

29 (1984) 423-424

W.D. McCuaig and M. Rosenfeld

Cyclability of r-regular r-connected graphs
$29(1984) \quad 1-11$ 
James B. McGuire and Colin J. Thompson

Distribution of iterates of first order difference equations

Iteration of piecewise linear maps on an interval

22 (1980) 133-143

D.K. McIl roy

A molecular model of ionic conductivity in nerve

24 (1981) 433-451

26 (1982) $285-316$

M. Megan

See P. Preda

Mihail Megan

See Petre Preda

Mihail Megan and Petre Preda

On exponential dichotomy in Banach spaces

23 (1981) 293-306

N.S. Mendelsohn

See F.E. Bennett

M. Mendès France and A.J. van der Poorten

Arithmetic and analytic properties of paper folding sequences

24 (1981) 123-131

Hans-Christian Mez

HNN-extensions of algebras and applications

$29 \quad(1984) \quad 215-229$

John Boris Miller

Representation of Boolean hypolattices

T.M. Mi $11 \mathrm{~s}$

See S.J. Goodenough

M. L. Mogra

Analytic mappings with negative coefficients in the unit disc

$29 \quad(1984) \quad 83-91$

M.L. Mogra and O.P. Juneja

$A$ radius of convexity problem

$24 \quad(1981) \quad 381-388$

S.P. Mohanty

See S.K. Shukla

B. Mond

Generalized convexity in mathematical programming

27 (1983) 185-202

S. Audinarayana Moorthy

Infinite series and the derived set of the aggregate

of the fractional parts of its partial sums

Infinite series and the derived set of the aggregate

21 (1980) 253-264

of the fractional parts of its partial sums: Addendum

Michael Moses

Recursive properties of isomorphism types [A]

25 (1983) 315-316

29 (1984) 419-421

R.N. Mukherjee

See Tanmoy Som

Bernd Mïller

Convergence tensor products and a strict topology

21 (1980) 281-301

Subhash Muttepawar

See Satya Deo 
Gerald Myerson and C.J. Smyth

On measures of polynomials in several variables:

Corrigendum

26 (1982) 317-319

S. Nababan and K.L. Teo

Existence and uniqueness of weak solutions of the

Cauchy problem for parabolic delay-differential

equations

21 (1980) 65-80

Itzhak Nada

See Abraham A. Klein

Subhashis Nag

Hyperbolic manifolds admitting holomorphic fiberings

26 (1982) 181-184

Sribatsa Nanda and Sudarsan Nanda

A nonlinear complementarity problem in Banach space

21 (1980) 351-356

Sudarsan Nanda

A note on a theorem on a nonlinear complementarity problem

See also Sribatsa Nanda

27 (1983) 161-163

P.P. Narayanaswami

See Stephen A. Saxon

K. Narsimha Reddy

Integral inequalities and applications

21 (1980) 13- 20

A. Nautiyal

See G.P. Kapoor

B.H. Neumann

Another single law for groups

Not quite inner automorphisms

See also Ann Chi Kim

23 (1981) 81-102

23 (1981) 461-469

M.N. Nkashama and M. Willem

Periodic solutions of the boundary value problem for the nonlinear heat equation

30 (1984) 99-110

Abu Salih Abdun Noor

Isotopes of nearlattices [A]

22 (1980) $472-474$

A.S.A. Noor

See William H. Cornish

Eric C. Nummela

The completion of a topological group

$21 \quad(1980) \quad 407-417$

Sheila Oates-Williams

Murskii's algebra does not satisfy $\mathrm{min}$

22 (1980) 199-203

Susumu Okada

The product of pre-Radon measures

25 (1981) 243-250

Yasuo Okuyama

On the absolute summability factor of Fourier series

$24 \quad(1981) \quad 327-337$

Hiroshi Onose

Aysmptotic behavior of nonoscillatory solutions of a

higher order functional differential equation

$24 \quad(1981) \quad 85-92$ 


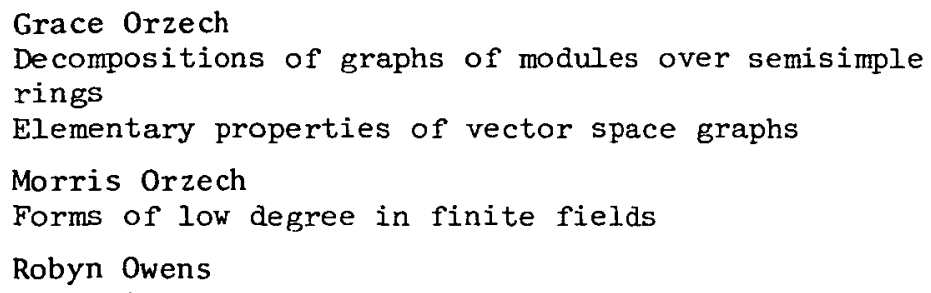

R. Parvatham and T.N. Shanmugam On analytic functions with reference to an integral operator

Ross Paull

Conically-similar viscous flows [A]

Maria Cristina Pedicchio

Closed structures on the category of topological

spaces determined by systems of filters

Franz Peherstorfer

Weight functions which admit Tchebycheff quadrature

Jean-Paul Penot

See Serge Gautier

\section{L.A. Peters}

Solutions of field equations in general relativity with spinor connection [A] $\begin{array}{lll}29 & (1984) & 405-413 \\ 30 & (1984) & 411-420 \\ 30 & (1984) & 45-58\end{array}$

29 (1984) 315-327

$28 \quad(1983) \quad 433-439$

$21 \quad(1980) \quad 477-478$

28 (1983) 393-400

28 (1983) 111-119

$26 \quad(1982) \quad 249-262$ 
Mario Petrich and Norma. R. Reilly

Varieties of groups and of completely simple

semigroups

23 (1981) 339-359

Ch.G. Philos

Oscillation of second order linear ordinary

differential equations with alternating coefficients

27 (1983) 307-313

Wilfred James Pinchin

Series solutions for the profiles of axisymetric

fluid-liquid interfaces [A]

27 (1983) 151-152

John Poland

Finite potent groups

$23(1981) \quad 111-120$

A.J. van der Poorten

$p$-adic methods in the study of Taylor coefficients

of rational functions

$29 \quad(1984) \quad 109-117$

See also M. Mendès France

See also Jeffrey D. Vaaler

Cheryl E. Praeger

Finite simple groups and finite primitive permutation groups

See also Janet A. Hoskins

28 (1983) 355-365

Govind Prasad

See Ashok Sahai

P. Preda and M. Megan

Exponential dichotomy of strongly discontinuous

semigroups

$30 \quad(1984) \quad 435-448$

Petre Preda and Mihail Megan

Nonuniform dichotomy of evolutionary processes in

Banach spaces

See also Mihail Megan

G. Prema and B.S. Kiranagi

On complete reducibility of module bundles

Geoff Prince

Toward a classification of dynamical symmetries in

classical mechanics

Geoffrey Eamonn Prince

Lie symmetries of differential equations and dynamical systems [A]

25 (1982) 309-311

Péter Pröhle

Does the Frobenius endomorphism always generate a

direct summand in the endomorphism monoids of fields

of prime characteristic?

$27 \quad$ (1983) $31-52$

28 (1983) 401-409

27 (1983) 53- 71

Frank P. Prokop

Pre-neighbourhood $\Lambda$-semi-lattices, neighbourhood lattices, and their associated continuous functions [A]

$24 \quad(1981) \quad 159-160$ 


\section{A.J. Pryde}

Spaces with homogeneous norms

Variational formulation of higher order elliptic

boundary value problems

$\begin{array}{lll}21 & (1980) & 189-205 \\ 28 & (1983) & 135-150 \\ 30 & (1984) & 289-293\end{array}$

Evegelos Psomopoulos

On the commutativity of torsion free rings

N.J. Pullman, H. Shank and W.D. Wallis

Clique coverings of graphs V: maximal-clique

partitions

$25 \quad(1982) \quad 337-356$

Barry G. Quinn

Fixed and random coefficient time series [A]

$24 \quad(1981) \quad 319-320$

Joshua H. Rabinowitz

Positivity notions for holomorphic line bundles over

compact Riemann surfaces

$23(1981) \quad 5-22$

T.G. Raghavan and I.L. Reilly

Uniformization of quasi-uniform spaces

23 (1981) 413-422

V. Raghavendra

A remark on an integral inequality

23 (1981) 195-197

S. Rajasekaran

See B.I. Bhatia

P.B. Ramanujan

See N.N. Chourasia

See B.C. Gupta

G. Vithal Rao

See M.L. Narayana Rao

K. Kuppuswamy Rao

See M.L. Narayana Rao

M. L. Narayana Rao, K. Kuppuswamy Rao and K. Satyanarayana

On Hering's flag transitive plane of order 27

25 (1982) 117-123

M. L. Narayana Rao and K. Satyanarayana

On a $C$-plane of order 25

$30 \quad(1984) \quad 27-36$

M.L. Narayana Rao, K. Satyanarayana and G. Vithal Rao

A class of translation planes of square order

$30 \quad(1984) \quad 59-66$

M. Rama Mohana Rao and P. Srinivas

Stability of functional differential equations of

Volterra type

$29(1984) \quad 93-100$

John M.S. Rassias

Weak and semi-strong solutions of the Schneider-

Tricomi problem in the Euclidean space; A uniqueness

theorem for the Chaplygin-Frankl problem: Corrigenda

Timothy R.C. Read

On choosing a goodness-of-fit test [A]

$23(1981) \quad 159-160$

G. Lakshma Reddy and K.S. Padmanabhan

On analytic functions with reference to the Bernardi

integral operator

$25 \quad(1982) \quad 387-396$ 
I. L. Reilly

See D.B. Gauld

See T.G. Raghavan

I.L. Reilly and M.K. Vamanamurthy

On hereditarily Lindelöf spaces

$21 \quad(1980) \quad 357-362$

Norman R. Reilly

See Mario Petrich

J-C. Renaud

Recurrence relations in a modular representation

algebra

$26 \quad$ (1982) 215-219

John Charles Renaud

On modular representation algebras of cyclic p-groups [A]

$\begin{array}{lll}28 & (1983) & 291-292 \\ 21 & (1980) & 237-252 \\ 28 & (1983) & 283-285\end{array}$

Michael von Renteln

Ideals generated by singular inner functions

J. Arias de Reyna

A connected metric space without an equally spaced chain of points

28 (1983) 283-285

A. H. Rhemtulla

See Ann Chi Kim

Akbar H. Rhemtulla

See Brian Hartley

N. Riahi

Nonlinear hydromagnetic convection in a moderate

Prandtl number fluid

Finite Prandtl number convection with nearly

insulating boundaries

23 (1981) 321-338

Gary D. Richardson

Applications of convergence spaces

R.W. Richardson

On orbits of algebraic groups and Lie groups

Conjugacy classes of involutions in Coxeter groups

24 (1981) 339-347

$21 \quad 1980) \quad 107-123$

25 (1982) $1-28$

26 (1982) $1-15$

M. Rimmer

Skew polynomial rings and skew power series rings [A]

21 (1980) 149-150

Russell Rimmer

Generic bifurcation from symmetric fixed points of

involutory area preserving maps [A]

21 (1981) $147-148$

Gerhard Ringel

See Brad Jackson

W.J. Robertson, I. Tweddle and R.E. Yeomans

On the stability of barrelled topologies, III

$22(1980) \quad 99-112$

V.K. Rohatgi

See R.G. Laha

M. Rosenfeld

See W.D. McCuaig 


\section{Fabio Rossi}

See Stefano Kasangian

\section{Bogdan Rzepecki}

An existence theorem for ordinary differential

equations in Banach spaces

Farrokh Saba

See David Burns

Ashok Sahai and Govind Prasad

Sharp estimates of approximation by some positive

linear operators

$30 \quad(1984) \quad 449-456$

Sundaram M.A. Sastry

See P. Kanniappan

A.V.V. Satyanarayana

See Prem Singh

K. Satyanarayana

See M.L. Narayana Rao

I.W. Saunders

Stochastic modelling in CSIRO: two examples

26 (1982) 143-155

R.B. Saxena

Degree of approximation of a function by Nörlund means

of its Fourier series

29 (1984) 13- 18

Stephen A. Saxon and P.P. Narayanaswami

Metrizable $(L F)$-spaces, $(d b)$-spaces, and the separable quotient problem

23 (1981) 65- 80

Michael G. Schooneveldt

Module structure in certain algebras [A]

$23(1981) \quad 395-412$

C.M. Scoppola

See A. Caranti

D.J. Scott and R.M. Huggins

On the embedding of processes in Brownian motion and the law of the iterated logarithm for reverse

martingales

P.R. Scott

Further inequalities for convex sets with lattice point constraints in the plane

A boundedness condition for sets with lattice point constraints

Michael John Sculley

The colloid physics of photosynthetic membranes [A]

R.J. Serven

See A.K. Boyle

S. Sessa

See M.S. Khan

S.M. Shah

See O.P. Juneja
27 (1983) 443-459

21 (1980) 7- 12

28 (1983) 5- 8

$23(1981) \quad 477-478$

21 (1980) $471-472$ 
H. Shank

See N.J. Pullman

T.N. Shanmugam

See R. Parvatham

S.D. Sharma

See R.K. Singh

P.W. Sharp

Unsteady waves on an open two layer fluid [A]

$27 \quad(1983) \quad 480$

S.K. Shukla, M.R. Sridharan and S.P. Mohanty

Stability of strongly regular graphs

23 (1981) 139-142

S.L. Shukla

See Vinod Kumar

J. Sichler

See M.E. Adams

Rafat Nabi Siddiqi

Generalized absolute continuity of a function of

Wiener's class

$22(1980) \quad 253-258$

Leon Simon

Boundary behaviour of solutions of the non-parametric

least area problem

Ivan Singer

Extension with larger norm and separation with double support in normed linear spaces

21 (1980) 93-105

Prem Singh and A.V.V. Satyanarayana

Subordination theorems for $p$-valent functions with

initial gaps

28 (1983) 121-129

R.K. Singh and S.D. Sharma

Non-compact composition operators

Composition operators and several complex variables

21 (1980) 125-130

23 (1981) 237-247

R.K. Singh and T. Veluchamy

Atomic measure spaces and essentially normal

composition operators

27 (1983) 259-267

Suresh Prasad Singh

On the degree of approximation by Szás z operators

24 (1981) 221-225

Donald K. Skilton

See Roger B. Eggleton

Bohdan Peter Smetaniuk

Topics in the theory of latin squares [A]

28 (1983) 443-444

Francis R. Smith

On the existence of embedded minimal 2-spheres in the 3-sphere, endowed with an arbitrary metric [A]

28 (1983) $159-160$

G.H. Smith

A note on complete hypersurfaces of non-positive

Ricci curvature

28 (1983) 339-342

Howard Smith

See C.J.B. Brookes 
William E. Smith

Efficiency of a university timetable. An application of entropy of choice

$\begin{array}{lll}30 & (1984) & 19-26 \\ 23 & (1981) & 49-63 \\ 28 & (1983) & 331-337 \\ 29 & (1984) & 349-356 \\ 23 & (1981) & 191-194 \\ 29 & (1984) & 39-46\end{array}$

C.J. Smyth

On measures of polynomials in several variables

See also Gerald Mverson

Anthony Sofo

Existence of limit cycles for a class of autonomous systems

Tanmoy Som and R.N. Mukherjee

Generalization of Leader's fixed point principle

D. Somasundaram

On a theorem of Brudno over non-archimedian fields

T.P. Speed

On the Möbius function of $\operatorname{Hom}(P, Q)$

M.R. Sridharan

See S.K. Shukla

P. Srinivas

See M. Rama Mohana Rao

V.K. Srinivasan

On some Gelfand-Mazur like theorems in Banach

algebras: On some Gelfand-Maxur like theorems in

$p$-normed algebras: Corrigenda

23 (1981) $479-480$

V.K. Srinivasan and Hu Shaing

On some Gelfand-Mazur like theorems in $p$-normed

algebras

$21 \quad(1980) \quad 211-221$

George Stamou

Characterizations of the sphere by the mean

II-curvature

$23(1981) 249-253$

Lawrence Stedman

Banach algebras with one dimensional radical

27 (1983) 115-119

Leon S. Sterling

Computing invariants for finitely presented nilpotent groups [A]

$24 \quad(1981) \quad 317-318$

B. Stockenberg

See H.-B. Knoop

A.N. Stokes

Continued fraction solutions of the Riccati equation

25 (1982) 207-214

John Strantzen

An average distance result in Euclidean $n$-space

$26(1982) \quad 321-330$

Anne Penfold Street

See Janet A. Hoskins

Deborah J. Street

Cyclotomy and designs [A]

$25 \quad(1982) \quad 473-474$

Ross Street

Notions of topos

23 (1981) 199-208 
Peter Summerside

Role of collective modes in some surface properties of metals [A]

$21 \quad(1980) \quad 317-320$

Cezary S. Surma

Discrete Green's functions and random resistive

networks [A]

$29 \quad(1984) \quad 139-140$

D.J. Sutton

Some problems in teletraffic with particular emphasis on limited availability networks [A]

$23(1981) \quad 317$

M. Swaleh

See M.S. Khan

Ferenc A. Szász

See Ismail A. Amin

G. Szekeres

The $n$-dimensional approximation constant

$29 \quad(1984) \quad 119-125$

Minoru Tanaka

On generalized Nörlund methods of summability II

22 (1980) 231-239

Limitation theorems for some methods of summability

22 (1980) $373-384$

A.B. Tayler

Differential equations and the real world

26 (1982) $421-443$

K.L. Teo

See K.G. Choo

See S. Nababan

U.B. Tewari and K. Parthasarathy

A theorem on abstract Segal algebras over some

commutative Banach algebras

Isomorphisms of some Segal algebras and their

multiplier algebras

25 (1982) 293-301

Walter Tholen

Note on total categories

25 (1982) 441-451

21 (1980) 169-173

Vidar Thomée

The finite difference versus the finite element method

for the solution of boundary value problems

$29 \quad(1984) \quad 267-288$

Colin J. Thompson

See James B. McGuire

Dale V. Thompson

See Saroop K. Kaul

H.B. Thompson

See J. Chabrowski

D.C. Trueman

Direct products of monogenic semigroups [A]

$24 \quad(1981) \quad 475-480$

Neil S. Trudinger and John I.E. Urbas

The Dirichlet problem for the equation of prescribed

Gauss curvature

On second derivative estimates for equations of MongeAmpère type

28 (1983) 217-231

$30 \quad(1984) \quad 321-334$ 
Leslie Ian Trudzik

Optimization in abstract spaces [A]

29 (1984) 141-143

Truong Công Nghê

Manifolds of smooth maps

Manifolds of smooth maps II: transversality

An inverse mapping theorem for Sobolev chains and its application

24 (1981) 1- 11

24 (1981) 49- 59

P.D. Tuan

See V.V. Anh

P.D. Tuan and V.V. Anh

Radii of convexity of two classes of regular functions

$21 \quad(1980) \quad 29-41$

Mihai Turinici

Stability criteria for contractive semigroups via

maximality procedures

27 (1983) 381-394

H. Tverberg

A generalization of Radon's theorem II

24 (1981) $453-469$

$24 \quad(1981) \quad 321-325$

I. Tweddle

See W.J. Robertson

M.H. Upham

Two-sided localization in semiprime FBN rings

$30 \quad(1984) \quad 179-191$

John I.E. Urbas

See Neil S. Trudinger

Alasdair Urquhart

Projective distributive $p$-algebras

Jeffrey D. Vaaler and A.J. van der Poorten

Bounds for solutions of systems of linear equations

24 (1981) 269-275

$25 \quad$ (1982) $125-132$

M.K. Vamanamurthy

See D.B. Gauld

See David B. Gauld

See I.L. Reilly

$T$. Veluchamy

See R.K. Singh

J. Vukman

A characterization of real and complex Hilbert spaces among all normed spaces

27 (1983) 339-345

Rudolf Výborný

Mean value theorems and a Taylor theorem for vector

valued functions

See also Jan Chabrowski

24 (1981) 69-77

Michel Waldschmidt

See Daniel Bertrand

G.E. Wall

Conjugacy classes in projective and special linear groups

W.D. Wallis

See N.J. Pullman 
Hwai-chiuan Wang

Growth of functions in $H^{P}$

25 (1982) $147-156$

Real Hardy spaces of an annulus

27 (1983) 91-105

Wang Lianxiang

See Xu Guangshan

Josephine A. Ward (nee Gooda11)

Banach spaces of pseudomeasures on compact groups with emphasis on homogeneous spaces [A]

22 (1980) 155-157

N.C. Weber

Rates of convergence for $U$-statistics with varying kernels

See also P.N. Kokic

Neville C. Weber

Martingale rates and weakly exchangeable arrays [A]

$22(1980) \quad 469-471$

Stanis law Wẹdrychowicz

See Józef Banaś

G.J. Weir and R.A. Wooding

Pressure transients in an idealised horizontal two

fluid reservoir

$25 \quad(1982) \quad 459-472$

Terence Weir

Generalized convexity and duality in mathematical programming [A]

21 (1980) 1- 5

James Wiegold

See J.L. Brenner

See Patrick Fitzpatrick

Ross Wilkinson

Semigroups in a topos and structure theory in inverse semigroups [A]

26 (1982) $471-472$

M. Willem

See M.N. Nkashama

C.S. Withers

A chain rule for differentiation with applications to multivariate Hermite polynomials

$30 \quad(1984) \quad 247-250$

R.A. Wooding

See G.J. Weir

Nicholas C. Wormald

Some problems in the enumeration of labelled graphs [A]

21 (1980) $159-160$

Z.S. Wu

See K.G. Choo

Zhan Sheng wu

On the optimal control of a class of hyperbolic systems [A]

26 (1982) $157-160$

$\mathrm{Xu}$ Guangshan and Wang Lianxiang

On explicit estimates for linear forms in the values

of a class of $E$-functions

$25 \quad(1982) \quad 37-69$ 
Rodney Ian Yager

On two variable p-adic $L$-functions [A]

25 (1982)

313

Sadayuki Yamamuro

Notes on the inverse mapping theorem in locally convex spaces

On a functional differential equation in locally convex spaces

On linear operators on ordered Banach spaces

On orthogonally decomposable ordered Banach spaces

Yuriko Yamamuro

Water waves above a sill [A]

Shinji Yamashita

Criteria for functions to be Bloch

Radii of univalence, starlikeness, and convexity

Beurling's ordinary value

Length and area inequalities for the derivative of a bounded and holomorphic function

21 (1980) 419-461

27 (1983) 269-283

27 (1983) 285-305

30 (1984) $357-380$

23 (1981) 157-158

$21 \quad(1980) \quad 223-227$

25 (1982) 453-457

28 (1983) 91- 99

M. Yamin

Relative relation modules of finite groups [A]

$30 \quad(1984) \quad 457-462$

23 (1983) $447-448$

Yang Lu and Zhang Jing-zhong

A generalisation to several dimensions of the

Neuberg-Pedoe inequality, with applications

Metric spaces which cannot be isometrically embedded

in Hilbert space

27 (1983) 203-214

30 (1984) 161-167

Adil Yaqub

See Hazar Abu-Khuzam

F.E. Yeomans

See W.J. Robertson

Katsuyuki Yoshikawa

On a 2-knot group with nontrivial center

$25(1982) \quad 321-326$

Norio Yosida

An oscillation theorem for sublinear elliptic

differential inequalities

David Yost

Average distances in compact connected spaces

$30 \quad(1984) \quad 387-394$

$26 \quad(1982) \quad 331-342$

A.I. Zahariev and D.D. Bainov

Oscillating properties of the solutions of a class of neutral type functional differential equations

$22(1980) \quad 365-372$

John Zeleznikow

On regular semigroups, semirings and rings [A]

$22(1980) \quad 313-314$

Zhang Jing-zhong

See Yang Lu

V. Zizler

Locally uniformly rotund renorming and decompositions of Banach spaces

$29 \quad(1984) \quad 259-265$ 